\title{
LIPOPROTEIN PATTERNS OF SERUM OBTAINED BY ZONE ELECTROPHORESIS
}

\author{
BY HENRY G. KUNKEL AND ROBERT J. SLATER
}

(From the Hospital of The Rockefeller Institute for Medical Research, New York, N. Y.)

(Submitted for publication February 25, 1952; accepted April 28, 1952)

For a number of years the lipoproteins of serum have been classified as alpha and beta types according to their electrophoretic mobility. Despite this designation very few studies of their electrophoretic properties have been reported. This is chiefly due to the fact that it is very difficult to determine exactly where the lipoproteins migrate when patterns of whole serum are obtained by the classical Tiselius method. Isolation of the lipoproteins can be achieved by chemical $(1,2)$ and ultracentrifugal methods $(3,4)$. However, even if turbidity and solubility problems in the electrophoretic separation of the isolated lipoproteins are overcome, some question remains as to whether the mobilities of these delicate complexes are the same in the isolated state as they are in whole serum. To circumvent these difficulties and to supplement the information obtained from the ultracentrifuge by Gofman, Lindgren and Elliott (3), Green, Lewis and Page (4), and Turner and associates (5) and that obtained from chemical fractionation by Lever and associates (2) and Russ, Eder and Barr $(6,7)$, the new technique of zone electrophoresis was applied to this problem. This method has the advantage that the electrophoretic components can be isolated directly so that lipid analyses can be carried out. The results obtained in the present study indicate that lipoprotein patterns can be obtained on whole serum and can be correlated in terms of relative electrophoretic mobility with the other proteins of serum.

\section{MATERIAL AND METHODS}

Sera were obtained from normal individuals and patients in the fasting state unless otherwise indicated. Analyses were carried out within three days after the removal of the serum except in the case of hypothyroidism where the serum had been stored at $4^{\circ} \mathrm{C}$ for 11 months without the development of significant sediment or lipid material at the surface. The patients with primary biliary cirrhosis were part of a group discussed previously (8). Total lipid, cholesterol and phospholipid analyses were carried out on the same alcohol-ether extract of serum or isolated protein fraction. The methods used were essentially those described in a previous report (8). Phosphorus was converted to phospholipid with the factor 25. In certain specifically designated cases cholesterol analyses on the multiple protein fractions were carried out by a simplified technique (9) where the color of the Liebermann-Burchard reaction was obtained directly for the mixture of free and esterified cholesterol in chloroform. Although this method was carefully standardized with the Sperry-Schoenheimer procedure, it was not as accurate as the latter because of variation in the esters which gave a slightly more intense color. It was used for the curves illustrated in Figures 3,4 and 5. For Figures 1 and 2 and Table I the conventional SperrySchoenheimer technique was used.

The method of zone electrophoresis was similar to that described previously (10). Filter paper and potato starch were used as the two types of supporting media in the present study. For the filter paper experiment, three to nine sheets of Whatman 3 MM paper 14 inches in length and 4 inches in width were superimposed on each other. A rectangular segment was cut out of the block of filter paper and the segment and block immersed in buffer solution. The wet paper was then dried to a barely moist state on another sheet of thick filter paper and the blocks placed on a silicone treated glass plate. Serum (2-5 cc.) was then applied to the cut segment of paper and this was replaced in its original position in the block of filter paper. Another glass plate was placed on top of the paper and clamps applied. Silicone grease was spread along the edge of the paper block between the plates to prevent evaporation at the sides. After placing the ends of the plates on the lips of perspex vessels containing buffer in contact with electrodes in a cold room, a current of 25-60 m.a. was applied through a variable voltage source of $100-500$ volts. The separation was carried out over a period of approximately 24 hours. The degree of separation could be followed by placing a light underneath the lower glass plate and observing the distance of migration of albumin which was readily visible at this high concentration of serum through the yellow bilirubin that was bound to it. Two other natural pigment bands in the $\beta$ globulin region also aided in determining the degree of separation. At the conclusion of the experiment after removing the glass plates the upper sheet was stained with brom phenol blue, cut into $1 / 2$ inch segments and the dye eluted to obtain a serum protein pattern (10). The lower block of paper sheets was also cut up into $1 / 2$ inch segments with special shears and placed in $20 \mathrm{cc}$. hot alcohol-ether $(3: 1)$ 
solution. The lipids were extracted over a ten hour period, the paper removed after pressing out the solvent, and the tubes centrifuged to remove precipitated protein or paper fragments. Aliquots of the clear alcohol-ether extract were then taken for cholesterol, phospholipid or total lipid analysis. The recovery of lipid from the segments was less than $100 \%$ because of removal of alcoholether with the paper. However, this error was relatively constant for each tube and was minimized by the excess of solvent.

Barbital buffer at $\mathrm{pH} 8.6, \mu 0.1$ was usually employed for the separation. Some barbital was extracted with the alcohol-ether but this did not interfere with the cholesterol or phospholipid analyses. However, total lipid carbon could not be determined because of barbital carbon. Barbital also interfered with nitrogen analyses on the fractions. Phosphate buffers were used where total lipid analyses were carried out. However, phospholipid analyses were difficult in this buffer and could only be carried out after washing the dried alcohol-ether residue with acid water to remove buffer phosphorus.

In the experiments where potato starch (Amend) was used as the supporting medium, a block of barely moist starch was used between glass plates in the same manner as filter paper. This was prepared by pouring a starch buffer mixture into a mold lined with wax paper. The starch was brought to a solid form by removing excess buffer with porous filter paper applied at the sides of the mold. The procedure will be described in detail elsewhere (11). The wax paper prevented sticking of the starch to the glass plates and could be peeled from the starch block readily at the conclusion of the experiment. Serum mixed with starch was placed in a rectangular space cut out of the starch block just as in the case of the filter paper block, or was applied directly with a pipette which penetrated into the starch. Contact at the electrode vessels was made with plastic sponges that pressed against the ends of the starch block. At the conclusion of the separation, the starch block was cut into segments with a thin spatula and the segments placed in test tubes. Saline was added to each tube $(1 \mathrm{cc}$.) and after stirring the starch and allowing it to settle, $0.05 \mathrm{cc}$. of supernate was removed from each tube. This was used to obtain a protein curve of the serum segments. The modified Folin-Ciocalteu procedure $(10,12)$ was found most suitable for this purpose. The starch did not contain material which interfered with this reaction. Ninhydrin and ultraviolet absorption curves could also be obtained but they required considerable washing of the starch to remove interfering material. Hot alcohol-ether was then added to the saline starch mixture or preferably to partially dried starch segments and the lipids extracted. Aliquots were taken for cholesterol and phospholipid analyses. Total lipid determinations were difficult because of some carbon material in the starch which was difficult to remove. Good recoveries could be obtained from the starch segments by further addition of solvent on a ground glass filter. The fresh solvent then displaced that mixed with the starch by suction. However, for most experiments a single extraction was used with a constant loss of solvent for each tube. Initial diffculties in the complete extraction of the cholesterol esters were overcome by longer extraction of the starch segments with hot solvent.

In the experiments where the lipoproteins were stained directly on filter paper after separation of the serum, the dried paper was immersed in a $50 \%$ ethanol solution saturated with Sudan III, placed at $37^{\circ} \mathrm{C}$ for three hours and washed with running water. Two superimposed filter paper strips were run together in these experiments and one strip, stained by the conventional method, was compared with the strip where only the lipid of the lipoprotein was stained.

Adsorption of proteins to the filter paper or starch was measured by letting buffer flow through the medium without an electric current. This was done by lowering the liquid level in one electrode vessel producing a siphonlike arrangement. The protein was carried along, leaving a trail if significant adsorption occurred. No separation of the serum proteins was obtained unless current was applied.

\section{RESULTS}

Preliminary observations on the localization of the lipoproteins relative to the other protein components of serum were carried out by simply staining the filter paper with Sudan III or other similar fat soluble dyes. Normal serum showed two distinct components, an intense spot in the $\beta$ globulin region and a more faintly staining spot in the albumin- $\alpha_{1}$ region. By careful comparison of the Sudan stained strips with an identical strip stained with brom phenol blue where all of the protein peaks were visible, it could be seen that the $\beta$ Sudan spot was close to the $\beta$ globulin peak but usually with a slightly lower mobility. The fainter Sudan spot did not correspond to the $\alpha_{1}$ peak but showed a slightly more rapid mobility. It lay partially under the albumin peak. Pathological sera with high lipid concentration showed many variations of the Sudan III patterns. Intense spots were visible in new positions on the pattern. Nephrotic sera usually showed a single spot moving in the region between the two main spots visible in the normal pattern. There appeared to be a general tendency for the normal lipid components to disappear and be replaced by new components, usually single, with a different mobility.

To test the validity of these observations and to determine whether the Sudan III was really staining the lipoprotein components selectively, 
direct lipid analyses were made on paper segments. To do this successfully it was necessary to increase greatly the capacity of the procedure so that $2-5 \mathrm{cc}$. of serum could be separated instead of $0.01 \mathrm{cc}$. as in the simple pattern stained with Sudan III. This was found possible by superimposing a number of sheets of filter paper, forming a block between glass plates. Figure 1 illustrates the protein pattern obtained by the brom phenol blue dye elution method in segments of the paper and the cholesterol and phospholipid patterns for the same segments. For this pattern $2.5 \mathrm{cc}$. of dialyzed serum were used with barbital buffer at $\mathrm{pH} 8.6, \mu 0.1$ and a current of 40 m.a. over a period of 24 hours in the cold room. Two major lipid peaks are visible, one slightly behind the $\beta$ globulin (designated $\beta$ ) and the other slightly behind the albumin but ahead of the $\alpha_{1}$ globulin (designated $\alpha$ ). This experiment confirms the localization of the lipoproteins observed with the Sudan staining. A third vague peak is visible extending from the origin to the $\beta$ peak. This vague area was also visible in the experiments with Sudan III. It has the appearance of a trail left behind as the $\beta$ component migrated from the origin. However, it is of interest that

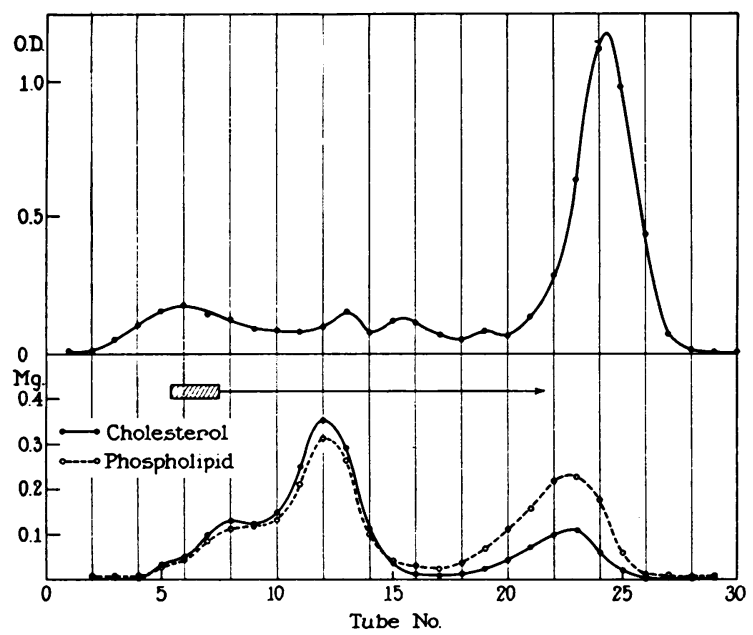

Fig. 1. Curves Showing the $\alpha$ and $\beta$ Lipoproteins for a Normal Serum Separated by Zone ElectroPhoresis in a Filter Paper Medium

The upper curve represents the protein pattern obtained by the brom phenol blue elution method, with O.D. referring to dye color. The lower curves represent the cholesterol and phospholipid values obtained in the same experiment on identical segments of paper.

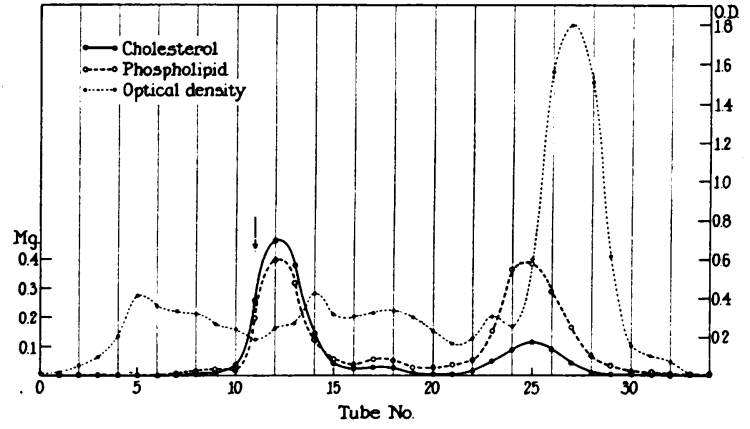

Fig. 2. Curves Showing the $\alpha$ and $\beta$ Lipoproteins for a Normal Serum Separated by Zone Electrophoresis in a Starch Medium

Protein, cholesterol and phospholipid analyses were carried out on the same starch segments. The optical density refers to the color intensity obtained with the modified Folin method of protein determination. The arrow signifies the origin.

total lipid analyses along with the cholesterol and phospholipid determinations indicated that this vague peak contained considerably more neutral fat than the $\beta$ or $\alpha$ peaks.

To further confirm the observations on the mobility of the lipoproteins relative to the other proteins of serum and to eliminate possible trailing of the $\beta$ component, a search was made for other supporting media in which to carry out the electrophoretic separation. Various materials were tried, glass beads and powders, resins, super cel and agar among others. All of these media caused considerably greater electroosmotic flow of water than did the paper (10) and the adsorption of the lipoproteins was considerable. Potato starch was finally settled on as the most satisfactory medium. No adsorption of the lipoproteins could be demonstrated. The electroosmotic flow was slightly greater than with the paper but not enough to detract from the usefulness of the starch. Lysozyme, another protein that was strongly adsorbed to filter paper in alkaline buffers, showed no detectable adsorption on the starch.

In Figure 2 the protein curve, determined by analyses with the modified Folin procedure on starch segments, is plotted along with cholesterol and phospholipid determinations for a different normal serum than that shown in Figure 1 . In general the agreement was good. The $\beta$ lipoprotein is in approximately the same position as in the separation on filter paper. The $\alpha$ lipopro- 
TABLE I

Cholesterol-phospholipid and free-total cholesterol ratios in the $\alpha$ and $\beta$ lipoprotein components of serum from five normal young males

The analyses were made on the tubes containing the highest concentration of cholesterol for each component.

\begin{tabular}{c|c|c|c}
\hline \multicolumn{2}{c|}{$\alpha$ Component } & \multicolumn{2}{c}{$\beta$ Component } \\
\cline { 2 - 3 } Chol/P lipid & F/T chol & Chol/P lipid & F/T chol \\
\hline 0.46 & 0.20 & 1.31 & 0.22 \\
0.62 & 0.21 & 1.36 & 0.25 \\
0.44 & 0.23 & 1.17 & 0.28 \\
0.60 & 0.25 & 1.21 & 0.29 \\
0.41 & 0.23 & 1.25 & 0.31 \\
\hline
\end{tabular}

tein migrated slightly slower in this experiment but it still was ahead of the $\alpha_{1}$ globulin, partially in the albumin fraction. A large number of determinations by the starch and filter paper procedures showed no essential difference in the relative positions of the $\alpha$ and $\beta$ lipoproteins by the two methods. The $\beta$ was always in the $\beta$ globulin position or slightly behind it while the $\alpha$ was always in the region between the $\alpha_{1}$ globulin and albumin peak. When large volumes of serum were used in the separation it was not always possible to observe the $\alpha_{1}$ globulin peak as clearly as in Figure 1 which represented an unusually good pattern. Protein curves were obtained by nitrogen analyses, ultraviolet absorption and by the ninhydrin technique as well as by the method mentioned. Certain differences were noted; however, regardless of the method of protein estimation, the $\alpha_{1}$ globulin peak was consistently observed along with the four other major peaks when barbital buffer ( $\mathrm{pH} 8.6, \mu 0.1)$ was used. In other buffers the $\alpha_{1}$ globulin peak was not seen. Experiments carried out in phosphate buffer at $\mathrm{pH} 7.6, \mu 0.1$, showed the $\alpha$ lipoprotein peak to fall right in the albumin fraction with the major peak sometimes slightly ahead of the albumin peak.

The major difference between the lipoprotein curve in Figures 1 and 2 was the almost complete absence of material with a slower mobility than the $\beta$ lipoprotein in the starch pattern. A slight peak is observed in Figure 2 near the origin but in other patterns on normal serum it was completely absent. Figure 2 also shows a small clearly defined component in the region between the $\alpha$ and $\beta$ lipoprotein peaks. This was seen occasionally in both the starch and filter paper in barbital buffer at $\mathrm{pH}$ 8.6. In addition to the absence of the trailing material, the starch system gave better recoveries and more uniform separation of the lipoproteins than did the filter paper.

In addition to defining the relative mobilities of the lipoproteins, Figures 1 and 2 demonstrate the low cholesterol to phospholipid ratio of the $\alpha$ lipoprotein as compared with the $\beta$. A similar relationship was found in 25 different normal sera that were studied. The phospholipid of the $\alpha$ peak was usually lower than the phospholipid of the $\beta$ component as in Figure 1 but an occasional serum was found to have the $\alpha$ phospholipid higher than the $\beta$. They are approximately equal in the normal serum of Figure 2. Determinations of cholesterol esters in the $\alpha$ and $\beta$ components showed that the $\mathrm{F} / \mathrm{T}$ ratio was consistently lower in the $\alpha$ fraction than in the $\beta$ (Table I). The trailing component visible in the filter paper experiments which was relatively high in neutral fat showed approximately the same cholesterolphospholipid ratio as did the $\beta$ lipoprotein.

Observations with acetate and phosphate buffers in a $\mathrm{pH}$ range from 5 to 7.6 gave results that were in general similar to those with barbital when the ionic strength was kept at 0.1 . Some separation of the $\alpha$ lipoprotein was sometimes seen and the $\beta$ lipoprotein gave a more diffuse peak; the basic division of the lipoproteins into two main groups continued to be evident. However, if the buffer concentration was reduced to an ionic strength of 0.05 , a definite subdivision of the main components was observed. In barbital buffer at

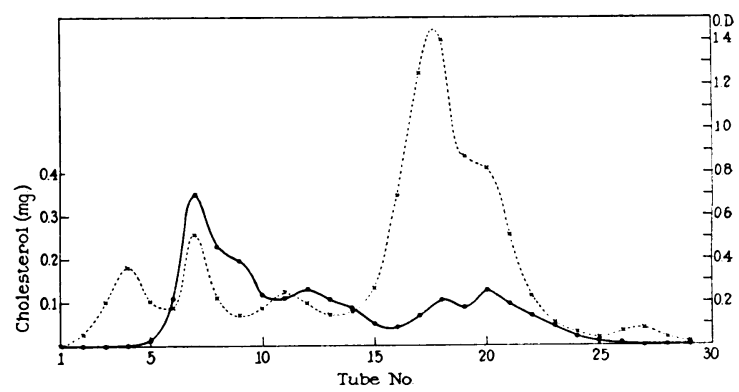

Fig. 3. Further Subdivision of the $\alpha$ and $\beta$ Lipoproteins Following Electrophoresis in a Starch Medium Using Phosphate Buffer pH 6.5, $\mu 0.05$

The cholesterol curve is shown (solid line) along with that for O.D. of protein color (dotted line). The $\beta$ lipoprotein peaks are in tubes 7, 9 and 12 ; the $\alpha$ peaks in tubes 18 and 20 . The origin was in tube 10 . 
$\mathrm{pH} 8.6, \mu 0.05$, the $\alpha$ component split up into two and sometimes as many as four fractions. The $\beta$ component always separated into at least two peaks. At a lower $\mathrm{pH}$, three $\beta$ fractions were consistently observed. Figure 3 illustrates the subdivision of the lipoproteins into at least five components at $\mathrm{pH} 6.5, \mu 0.05$. The splitting of the albumin peak at this $\mathrm{pH}$ was an unexpected finding and is of interest in other connections. The high cholesterol peak in tube 7 was observed during the course of the experiment as a sharp orange colored band. It was peculiar in that the band was narrower than the origin and of such intensity of color that a grouping together of lipoprotein particles must have occurred, carrying pigment with them. A surprisingly high lipid concentration was always found in this band. The lipemic particles of non-fasting serum also moved in this band. At lower $\mathrm{pH}$ levels in acetate buffers the colored band was also visible but migrated in an intermediate position among the other lipoprotein peaks. This phenomenon probably resulted from boundary anomalies due to the high protein to buffer ratios.

\section{Pathological sera}

Lipoprotein curves were obtained for a number of pathological sera employing the filter paper procedure. The adsorption and trailing of the $\beta$ lipoprotein was a greater problem, particularly with lipemic sera, than it was with the normal sera mentioned above. However, again total lipid analyses indicated that the trailing component was high in neutral fat. To avoid this problem the starch medium with barbital buffer $\mathrm{pH} 8.6$, $\mu 0.1$ was adopted for the main observations on high lipid sera. Figure 4 illustrates the lipoprotein curves obtained for three different patho-

TABLE II

Results of lipid determinations on the' whole sera used in Figures 4 and 5

\begin{tabular}{l|c|c|c}
\hline \hline & Total lipid & Cholesterol & Phospholipid \\
\cline { 2 - 3 } & 691 & 220 & 260 \\
Normal (Fig. 4) & 1,230 & 380 & 610 \\
Xanthoma & 1,397 & 452 & 375 \\
Hypothyroid & 1,532 & 550 & 522 \\
Essential Hyper- & & & \\
$\quad$ cholesterolemia & 2,741 & 733 & 411 \\
Nephrosis A & 805 & 282 & 320 \\
Nephrosis B & & \\
\hline
\end{tabular}

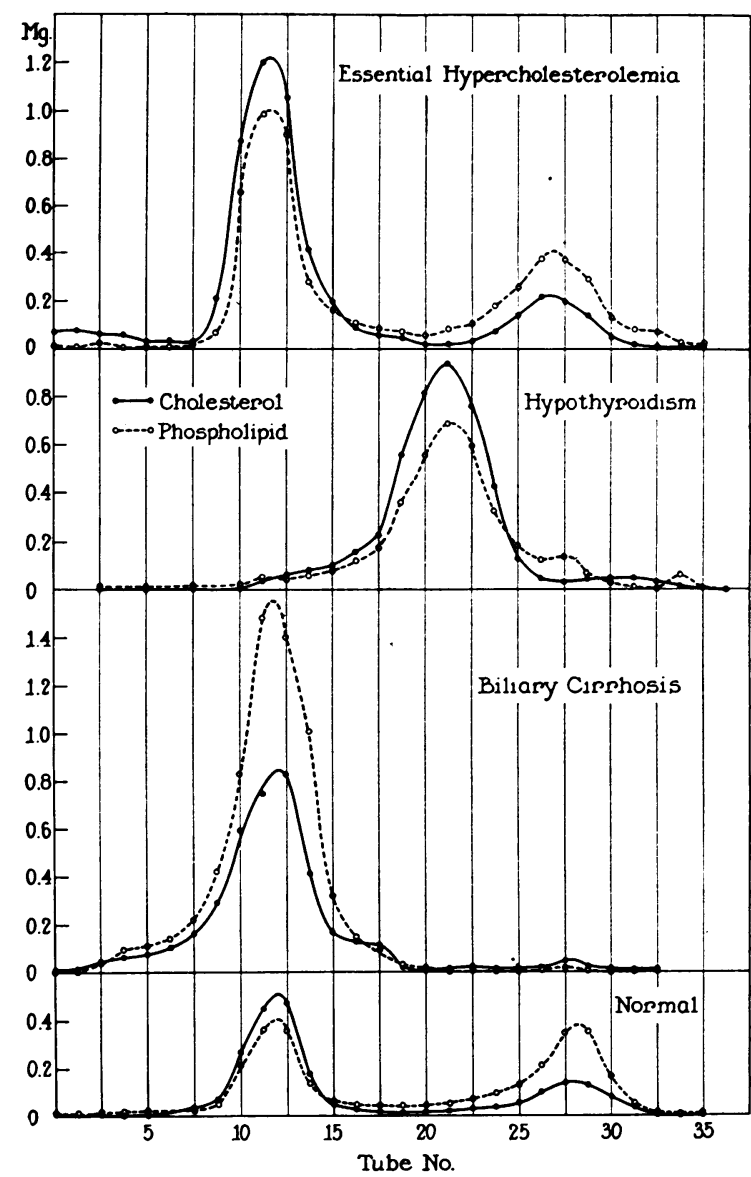

Fig. 4. Lipoprotein Patterns for Three Pathological Sera Contrasted with That of a Normal Serum Employing a Similar Degree of Separation in Each Case

The origin was in tubes 8 and $9 ; 2.5 \mathrm{cc}$. of each serum were used, $\mathrm{pH} 8.6, \mu 0.1$.

logical sera and a normal serum where the colored albumin band was permitted to migrate the same distance from the origin in each case. The marked variation from the normal is apparent.

Table II furnishes data on the lipid composition of these sera. The pattern for the biliary cirrhosis serum is characteristic for this disease and is similar to that observed in the serum of four other patients. A very high peak consisting predominantly of phospholipid migrated at approximately the same relative mobility as normal $\beta$ lipoprotein. The $\alpha$ lipoprotein was extremely low. In two of the five sera it was completely absent. In one case the main peak was notched indicating partial splitting of this component. In addition to the five high lipid sera from patients 


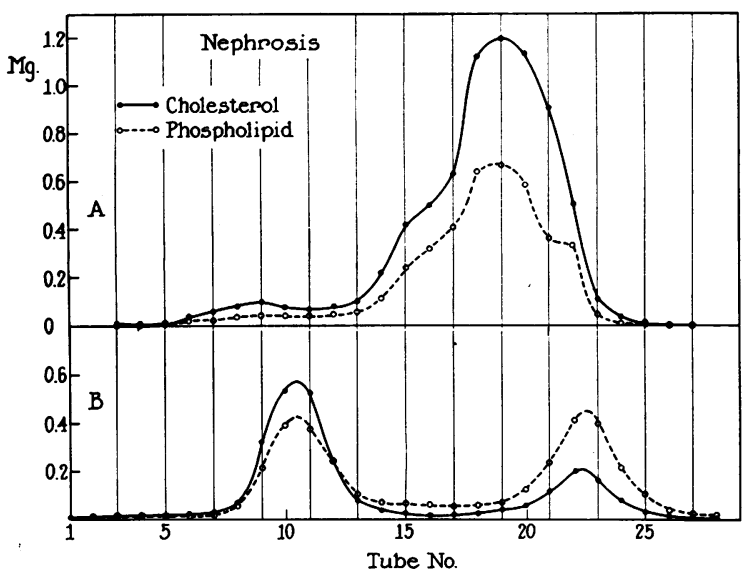

Fig. 5. Lipoprotein Patterns Obtained in a Patient With Nephrosis, Before (A) ANd After a Spontaneous Diuresis (B)

The origin was in tubes 8 and 9, with a similar degree of separation in each case.

with biliary cirrhosis, two sera were examined from patients with this condition who at one time had lipid abnormalities but who despite continuation of jaundice developed levels in the low normal range. These sera showed a pattern similar to that just described with almost complete absence of the $\alpha$ lipoprotein peak and all the lipid in a component resembling the normal $\beta$ lipoprotein in terms of relative mobility. Two sera were also obtained from patients with obstructive jaundice due to a stone in one case and carcinoma of the head of the pancreas in the other. These sera also showed a similar pattern with a disappearance of the $\alpha$ lipoprotein component.

The pattern from the serum of the patient with hypothyroidism also shows a low $\alpha$ lipoprotein peak and little or no lipid in the position of the usual $\beta$ component. The one large component has an intermediate mobility. This was the only hypothyroid pattern available so that it is impossible to say that it was characteristic of this disease.

Three sera were examined from individuals with essential hypercholesterolemia; two were of the familial type. None of these individuals had any real complaints. One showed xanthelasma. The pattern in Figure 4 is typical of this group. The $\alpha$ lipoprotein was normal or moderately depressed while the $\beta$ lipoprotein was considerably increased, slightly more for the cholesterol than for the phospholipid. No components with abnormal mobilities were observed.

Four sera were examined from children with nephrosis. Considerable variation was observed but in general one large irregular peak consisting of from one to three components was observed in the region between the $\alpha$ and $\beta$ peaks of normal serum. The $\beta$ peak was always almost completely absent. The $\alpha$ peak appeared markedly reduced but because of overlap by the main abnormal component it was sometimes difficult to differentiate. Figure 5A illustrates a pattern obtained in one of the patients at a time when edema, ascites and severe albuminuria were present. The patient developed a diuresis, probably spontaneously, with complete loss of retained fluid, a decrease in the albuminuria, and a gain in body weight. A second pattern obtained seven months after the first is shown in Figure $5 \mathrm{~B}$, with the same degree of separation. The second pattern is essentially normal.

\section{DISCUSSION}

Many of the observations mentioned above, particularly those concerning the composition of the $\alpha$ and $\beta$ lipoproteins, have been reported previously $(2,6)$, from experiments where the lipoproteins were isolated by the methods of Lever and associates (2). It is of interest that most of the results for normal serum are in approximate agreement despite the basic differences in the methods of chemical and electrophoretic separation. The normal lipoproteins certainly can be divided into the two main classes, the $\beta$ type with a cholesterol phospholipid ratio of approximately 1.3 and the $\alpha$ type with a ratio of approximately 0.5. The finding of a consistently lower freetotal cholesterol ratio in the $\alpha$ lipoprotein than in the $\beta$ demonstrates a further difference.

Of some interest is the finding that the $\alpha$ lipoprotein migrates slightly ahead of the $\alpha_{1}$ globulin component and falls partially in the albumin fraction of serum. In phosphate buffer at $\mathrm{pH} 6.5$ it actually migrated partially ahead of the major albumin peak. Attempts have been made to correlate the $\alpha_{1}$ globulin area in free electrophoresis with the concentration of $\alpha$ lipoprotein determined by other methods. These have not been particularly successful and perhaps the slightly greater mobility of the lipoprotein is the explanation. It 
should be mentioned that in free electrophoresis experiments the lipid of the lipoproteins contributes to the refractive index increment of the proteins while in the present experiments the lipid was not estimated by either the dye elution or the modified Folin procedure. This accounts for some of the differences in the protein curves obtained by the two types of electrophoresis discussed previously (10). The slightly slower mobility of the $\beta$ lipoprotein compared to the $\beta$ globulin peak may be explained in this way; and, if the lipid were measured as in free electrophoresis experiments, these two peaks might fuse. A similar explanation might apply to the $\alpha_{1}$ globulin peak although here the influence of the lipid would be less marked.

A large scale quantitative analysis of the normal fractions, such as that carried out by Russ, Eder and Barr (6) with their striking results of sex and age differences, is more practically done by the chemical methods. The results with the zone electrophoresis method certainly confirm the validity of the chemical division for such relatively normal sera. For pathological sera with large lipid alterations the results of the chemical method are more difficult to interpret and do not correlate as well with the results of zone electrophoresis. Some of the pathological sera examined in the present study, particularly those from patients with nephrosis, showed the major lipoprotein to have an intermediate mobility clearly different from the $\alpha$ and $\beta$ components of normal serum. By the chemical fractionation procedure this abnormal component falls chiefly in the same fraction with the normal $\beta$ lipoprotein. Sera from patients with biliary obstruction, intrahepatic or extrahepatic in type, were studied particularly. Of nine such sera from different patients, not one showed a significant amount of the normal $\alpha$ component; it was either entirely absent or present in very low concentrations. This was a general phenomenon applying to other high lipid pathological states; in no case was an increase in the $\alpha$ component observed. Three patients with biliary obstruction examined by the chemical procedure have been reported (7) to have an increase in the fraction showing the $\alpha$ component of normal serum. These results are difficult to understand; perhaps the high phospholipid content of the lipo- protein of these sera alters their solubility so that they fall into the $\alpha$ fraction or perhaps the high levels of bile salts are responsible. For these pathological sera the method of zone electrophoresis would appear to possess certain advantages, particularly in demonstrating new components.

Although the procedure utilizing filter paper as the stabilizing medium showed adsorption of the $\beta$ lipoprotein in the path of migration, it furnished some information not provided by the starch system. The trailing material showed a high neutral fat content relative to that of the main $\beta$ component. Such a selective adsorption of the larger lipoprotein molecules containing increased amounts of neutral fat might be expected and these molecules probably correspond to the higher Sf components described by Gofman, Lindgren and Elliott (3). Of particular interest is the fact that the cholesterol-phospholipid ratio of the adsorbing component was very close to that of the $\beta$ lipoprotein. This, together with the fact that the high neutral fat material had the same mobility as the $\beta$ component when the nonadsorbing starch medium was used, suggests a close relationship.

The low cholesterol-phospholipid ratio obtained for the $\beta$ component in the sera of patients with biliary obstruction differs strikingly from the composition of the $\beta$ lipoprotein in normal serum. It may be that this represents an entirely different lipoprotein that just happens to migrate with the mobility of the normal component. However, it seems more likely that this protein is related to the normal $\beta$ lipoprotein with a slightly different lipid composition. Isolation of this lipoprotein in the ultracentrifuge in preliminary experiments has indicated that it has a lipid-protein ratio close to but slightly higher than the normal $\beta$ lipoprotein.

\section{SUM M ARY}

1. Separation of $\alpha$ and $\beta$ lipoproteins of normal serum into two main fractions was readily possible by zone electrophoresis in a filter paper or starch medium containing barbital buffer. Lipoprotein curves were obtained and the relative mobilities of the components correlated with the mobilities of the other serum proteins.

2. The cholesterol-phospholipid ratios of the two major components showed approximate 
agreement with the results obtained by other workers by chemical fractionation. A lower freetotal cholesterol ratio was found in the $\alpha$ fraction than the $\beta$.

3. Further subdivision of the $\alpha$ and $\beta$ lipoproteins was obtained with low ionic strength buffers in the starch medium. The $\alpha$ lipoproteins consistently separated into two and sometimes three fractions. The $\beta$ lipoproteins separated into at least three fractions, one of which could be seen as a sharp orange band.

4. Marked variations from the normal pattern were found in pathological sera with elevated lipid concentrations. These were characterized by a general tendency of diminution or disappearance of the $\alpha$ lipoproteins and either an increase of the $\beta$ type or the appearance of a large component with an abnormal mobility.

\section{ACKNOWLEDGMENT}

The senior author is indebted to Professor Arne Tiselius for valuable suggestions in the early portion of this work which was carried out at the Institute of Biochemistry, Uppsala, Sweden.

\section{REFERENCES}

1. Oncley, J. L., Gurd, F. R. N., and Melin, M., Preparation and properties of serum and plasma proteins. XXV. Composition and properties of human serum $\beta$-lipoprotein. J. Am. Chem. Soc., 1950, 72, 458.

2. Lever, W. F., Gurd, F. R. N., Uroma, E., Brown, R. K., Barnes, B. A., Schmid, K., and Schultz E. L., Chemical, clinical, and immunological studies on the products of human plasma fractionation. XXL. Quantitative separation and determination of the protein components in small amounts of human plasma. J. Clin. Invest., 1951, 30, 99.

3. Gofman, J. W., Lindgren, F. T., and Elliott, H., U1tracentrifugal studies of lipoproteins of human serum. J. Biol. Chem., 1949, 179, 973.

4. Green, A. A., Lewis, L. A., and Page, I. H., A method for the ultracentrifugal analysis of $\alpha$ and $\beta$ serum lipoproteins. Federation Proc., 1951, 10, 191.

5. Turner, R. H., Snavely, J. R., Goldwater, W. H., Randolph, M. L., Sprague, C. C., and Unglaub, W. G., The study of serum proteins and lipids with the aid of the quantity ultracentrifuge. I. Procedure and principal features of the centrifugate of untreated normal serum as determined by quantitative analysis of samples from ten levels. J. Clin. Invest., 1951, 30, 1071.

6. Russ, E. M., Eder, H. A., and Barr, D. P., Proteinlipid relationships in human plasma. I. In normal individuals. Am. J. Med., 1951, 11, 468.

7. Barr, D. P., Russ, E. M., and Eder, H. A., Protein lipid relationships in human plasma. II. In atherosclerosis and related conditions. Am. J. Med., 1951, 11, 480.

8. Ahrens, E. H., Jr., Payne, M. A., Kunkel, H. G., Eisenmenger, W. J., and Blondheim, S. H., Primary biliary cirrhosis. Medicine, 1950, 29, 299.

9. Kingsley, G. R., and Schaffert, R. R., Determination of free and total cholesterol by direct chloroform extraction. J. Biol. Chem., 1949, 180, 315.

10. Kunkel, H. G., and Tiselius, A., Electrophoresis of proteins on filter paper. J. Gen. Physiol., 1951, 35, 89.

11. Kunkel, H. G., and Slater, R. J., Zone electrophoresis in a starch medium. Proc. Soc. Exper. Biol. \& Med., in press.

12. Lowry, O. H., Rosebrough, N. J., Farr, A. L., and Randall, R. J., Protein measurement with the Folin phenol reagent. J. Biol. Chem., 1951, 193, 265. 